\title{
Platelet-rich plasma inhibits Adriamycin-induced inflammation via blocking the NF-KB pathway in articular chondrocytes
}

\author{
Haijun Zhao ${ }^{*} \mathbb{D}$, Weijie Zhu, Wude Mao and Chengkai Shen
}

\begin{abstract}
Background: Previous studies showed that doxorubicin could lead to osteoarthritis (OA) by inducing chondrocyte inflammation and apoptosis. Besides, it is reported that platelet-rich plasma (PRP) could suppress the activation of inflammatory NF-KB signaling. Here, we aimed to determine whether PRP was able to exert a protective effect against doxorubicin-induced chondrocyte damages.

Methods: To determine whether PRP protects chondrocytes against destabilization of the medial meniscus (DMM)induced osteoarthritis, mice were treated with PRP and doxorubicin, and the cartilage destruction was observed through Safranin O-fast green staining and osteoarthritis scoring. ELISA assay was used to check the release of TNF-a and ILs. In vitro, we treated chondrocytes with doxorubicin and PRP; CCK-8 was used to measure cell viability. Western blot, real-time PCR, and ELISA were applied to check apoptosis-related signaling and inflammation-associated factors.

Results: The results from the mouse model suggested that PRP attenuated doxorubicin-induced cartilage destruction in vivo. Doxorubicin promoted chondrocyte apoptosis while PRP ameliorated this damage. PRP inhibited doxorubicin-induced dysregulation of cell matrix-related factors, including SOX9, Col2A1, Col10A1, and Aggrecan, reduced protein levels of doxorubicin-induced inflammatory markers, COX-2, and iNOS, and blocked doxorubicin-induced phosphorylation of IKB and NF-kB in articular chondrocytes.
\end{abstract}

Conclusions: PRP improved doxorubicin-induced damage on chondrocytes. This research might provide a new theoretical basis for the clinical treatment of osteoarthritis caused by doxorubicin.

Keywords: PRP, Doxorubicin, Osteoarthritis, Chondrocyte, Inflammation, NF-kb

\section{Introduction}

Osteoarthritis $(\mathrm{OA})$ is a prevalent joint disorder globally, occurring in about $20 \%$ of people aged over 60 -year old, and severely affecting the daily life of those patients (Chen et al. 2017). During the process of OA, cartilage destruction usually occurs in joints of knees, hands, and spine, slowly damaging articular cartilage and disabling the function of whole joints (Sherwood 2019). The

*Correspondence: HaijunZhaoJiaozhou@163.com

Department of Joint Trauma Surgery, Qingdao Jiaozhou Central Hospital, No. 29 Xuzhou Road, Jiaozhou 266300, Shandong, People's Republic of China crucial pathological factors of OA involve aging, obesity, joint instability, and especially joint inflammation (Sherwood 2019). It is suggested that inflammation functions at the early stage of OA drive various pathological changes during its progression, including articular cartilage degeneration (Robinson et al. 2016). Articular cartilage only contains one type of cells, namely chondrocytes, filled with the extracellular matrix that mainly constructed by collagens (Liao et al. 2017; Guilak et al. 2018). It was indicated that chondrocytes undergo apoptotic cell death during OA pathogenesis, and the collagens could be degraded by matrix metalloproteinases (MMPs), such as MMP3 and MMP13, which were upregulated in OA 
samples as reported in previous studies (Lepetsos et al. 2019). These studies indicated that targeting cell apoptosis and matrix remodeling might be effective strategies for OA therapy.

Despite the high prevalence of $\mathrm{OA}$ and its severe impacts on human health, no treatment has been approved by FDA to prevent or ameliorate OA progression, and surgical joint replacement is the only effective strategy for end-stage OA (Bannuru et al. 2015; Gregori et al. 2018). Hence, it is urgent to develop novel and effective therapeutic manners for OA. Platelet-rich plasma (PRP) is autologous plasma with concentrated platelets, which would secrete various growth factors, cytokines, chemokines, and other functional molecules when activated (Xie et al. 2014). In recent years, an increasing number of studies have focused on PRP application to ameliorate the inflammatory state during $\mathrm{OA}$ (Xu et al. 2017; Fukawa et al. 2017; Fotouhi et al. 2018; Chouhan et al. 2019). Fotouhi et al. (2018) reported that intra-articular PRP injections showed multiple favorable results for patients with OA. Another study on the guinea pig model of knee OA suggested that multiple PRP injections provided better therapeutic outcomes than a single injection (Chouhan et al. 2019). Here, we tried to further elucidate the function of PRP for OA treatment.

Doxorubicin is an effective antibiotic drug with a broad anti-tumor spectrum, although its clinical application is limited by multiple side effects, including generating reactive oxygen species and nitric oxide and causing cell apoptosis (Kalivendi et al. 2001; Mukhopadhyay et al. 2009; Speth et al. 1988; Rivankar 2014). Kumagai et al. (2012) also proposed that doxorubicin could induce apoptosis of chondrocytes by causing cell volume decrease. Yet, the profound mechanisms underlying doxorubicin-induced apoptosis of chondrocytes and whether PRP administration could ameliorate this doxorubicincaused damage are still unclear.

In this study, we performed in vitro and in vivo experiments to evaluate whether PRP was able to reverse doxorubicin-induced damage on chondrocytes, with the hope to provide novel insights into developing OA therapies.

\section{Materials and methods}

\section{Animal model}

All animal experiments in this study were conducted strictly following the legislation of the use and care of laboratory animals in China and approved by Qingdao Jiaozhou Central Hospital (No. 632278\#ERF). 12-weekold male C57BL/6 mice were housed in a designated mouse facility operating at a controlled temperature $\left(24{ }^{\circ} \mathrm{C}\right)$ and a circadian rhythm of $12 \mathrm{~h}$ and had free access to clean water and foods. These mice were randomly divided into the indicated groups in corresponding experiments. OA was induced in mice by destabilizing the medial meniscus (DMM) of the knees. One week after surgery, the mice received doxorubicin $(10 \mathrm{mg} / \mathrm{kg})$ injection and/or intra-articular injection of $15 \mu \mathrm{L}$ PRP every week. After 4 weeks, the mice were euthanized, and the knee joints and plasma were collected ( $\mathrm{Hu}$ et al. 2020).

\section{PRP extraction}

PRP was isolated from the blood of euthanized mice. In brief, whole blood was collected using heparin-containing sterile syringes and centrifuged for $10 \mathrm{~min}$ at $1800 \mathrm{rpm}$. The bottom layer with red blood cells was discarded, and the upper and intermediate layers were collected and centrifuged again at $3000 \mathrm{rpm}$ for $6 \mathrm{~min}$. After carefully removing the upper $75 \%$ supernatant, the remainder was resuspended in DMEM/F12 media and filtered (Bos-Mikich et al. 2018) to obtain PRP. PRP concentration was determined according to TGF- $\beta 1$ concentration via ELISA (Additional file 1: Fig. S1).

\section{Enzyme-linked immunosorbent assay (ELISA)}

The serum levels of inflammatory factors TGF- $\beta 1$, TNF$\alpha$, IL- $1 \beta$, and IL- 6 were checked using ELISA following the manufacturer's instructions. IL-6 (M6000B), TGF$\beta 1$ (DB100B), TNF- $\alpha$ (MTA00B), IL-1 $\beta$ (MLB00C) were purchased from R\&D systems (Minneapolis, MN).

\section{Histopathologic analysis}

The knee joint samples were collected and fixed in $4 \%$ paraformaldehyde for $24 \mathrm{~h}$. The bones were placed in $10 \%$ EDTA for 1 month to perform decalcification, embedded with paraffin, prepared as $5 \mu \mathrm{m}$ thick sections, and stained with Safranin O-fast green staining kit (G1371, Solarbio, China) following manufacturer's instruction. The images were captured and calculated. The scores of the femur and tibia were summed and presented as the OARSI score for each sample.

\section{Cell culture}

Mice were sacrificed, and the total articular cartilage was isolated from the femur head, cut into pieces, and digested with $1.5 \mathrm{mg} / \mathrm{mL}$ pronase for $2 \mathrm{~h}$ and collagenase II under shaking at $37^{\circ} \mathrm{C}$ for $8 \mathrm{~h}$. Cells were then filtered through a $70 \mu \mathrm{m}$ cell strainer and washed 3 times with PBS. Subsequently, the obtained chondrocytes were placed in culture plates and maintained in DMEM/ F12 media (Gibco, Thermo, MA, USA) containing 10\% fetal bovine serum (FBS, Gibco), $100 \mathrm{U} / \mathrm{mL}$ penicillin, and $100 \mu \mathrm{g} / \mathrm{mL}$ streptomycin. The media were changed every 2 days. After preparation of chondrocytes from joint articulation, toluidine blue staining and collagen type II immunohistochemical staining were carried out 
to identify the purity of these chondrocytes (Additional file 1: Fig. S1).

Bone marrow-derived macrophages (BMMs) were extracted from the bone marrow of the femur and tibia of mice and cultured in Dulbecco's modified Eagle medium (DMEM) supplemented with 10\% FBS with M-CSF $(10 \mathrm{ng} / \mathrm{mL})$. BMMs were differentiated in a humidified chamber at $37^{\circ} \mathrm{C}$ with $5 \% \mathrm{CO}_{2}$ with changing cell culture media every 3 days until they became confluent. Afterward, cells were plated for experimental treatments.

ATDC5 cells were cultured in Alpha modification of Eagle's media containing 10\% FBS, $100 \mathrm{U} / \mathrm{mL}$ penicillin, and Insulin-Transferrin-Selenium solution. All cells were cultured at $37^{\circ} \mathrm{C}$ in a humidified incubator with $5 \% \mathrm{CO}_{2}$.

\section{Cell viability assay}

The proliferation ability of cardiomyocytes was analyzed by CCK- 8 kit. In brief, cells were digested and plated into 96-well plates with a density of $1 \times 10^{3}$ cells per well overnight and treated with doxorubicin or PRP at the indicated concentration for $24 \mathrm{~h}$. At the treatment, $10 \mu \mathrm{L}$ of CCK-8 (Roche, Basel, Switzerland) was added to each well, and cells were continuously incubated for 1 more hour at the incubator. Then the absorbance values at $450 \mathrm{~nm}$ was detected by an absorbance reader.

\section{Western blot}

Cells were lysed in $1 \times$ ice-cold RIPA lysis buffer (Beyotime, Shanghai, China) and centrifuged to discard the deposition. The protein concentration was determined using BCA kits (TakaRA, Dalian, China). A total of $35 \mu \mathrm{g}$ proteins were separated on $10 \%$ SDS-polyacrylamide gel (PAGE) and transferred to polyvinylidene difluoride (PVDF) membranes. The membranes were blocked for $1 \mathrm{~h}$ at room temperature with $5 \%$ nonfat milk dissolved in $1 \times$ TBST and incubated with specific primary antibodies at $4{ }^{\circ} \mathrm{C}$ overnight. Then the membranes were further incubated for $2 \mathrm{~h}$ at room temperature with the corresponding secondary antibodies. After that, labeled proteins were visualized by an ECL kit (Invitrogen, CA, USA) and captured using Gel imaging system (BD, USA). Primary antibodies against p-NF- $\kappa B$ (3033), NF- $k B$

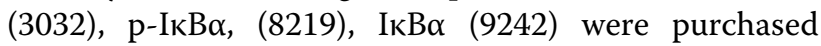
from Cell Signaling Technology (MA, USA) and against $\beta$-actin (sc-1616), COX-2 (sc-376861), Sox9 (SC-166505), iNOS (SC-7271), PARP (SC-74470) and cleaved PARP (SC-56196) were purchased from SantaCruz Biotechnology (CA, USA) and diluted following the manufacturers' protocols.

\section{RNA extraction and real-time PCR}

Total RNAs were extracted using TRIzol Reagent (Invitrogen, CA, USA) following the manufacturer's protocols after indicated treatments. Their concentration was determined spectrophotometrically at $260 \mathrm{~nm}$ (Thermo-Scientific NanoDrop 2000), and their quality and purity were verified by A260/A280 ratio (1.82.2). Then RNAs were reverse transcribed to cDNA using a cDNA Synthesis Kit (Takara Biotechnology, Japan). Quantitative real-time PCR (qRT-PCR) was performed using the SYBR Green SuperMix kit (BD, USA) to detect gene expression following the manufacturer's protocols. Small endogenous nucleolar U6 snRNA and GAPDH were used as the internal controls for normalizing miR-125a-5p and MTFP1, respectively. Gene expression level was determined using the $2^{-\Delta \Delta C t}$ method. The primers used in this study were MMP1 forward 5'-ATACGTTGGTGGTGTTGTAATGT-3' and reverse 5'-GTCA CCTCTTTGGATGCCATAA A-3', MMP3 forward, 5'-TTAAAGACAGGCACTTTT G GCG-3' and reverse 5'-CCCTCGTATAGCCCAGAA CT-3', MMP13 forward 5'-CTT TGGCTTAGAGGT GACTGG-3' and reverse 5'-AGGCACTCCACATCT TGGTTT-3', TNF- $\alpha$ forward 5'-TGGAACACGTCG TGGGATAATG-3' and reverse 5'-GGCAGAC TTT GGATGCTTCTT-3', IL-6 forward 5'-GGCGGATCG GATGTTGTGAT-3' and reverse 5'-GGACCCCAG ACAATCGGTTG-3', Col2A1 forward 5'-CCTCAA GGCA AAGTTGGTCCT-3' and reverse 5'-CTCCCG TCTCACCGTCTTTT-3', Col10A1 forward 5'-GGT GTGAATGGGCGGAAAG-3' and reverse $5^{\prime}$-GCT TCCCAATACCTT CTCGTC-3', as well as Aggrecan forward 5'-CCCAGGATAAAACCAGGCAG-3' and reverse 5'-CGGCCAAGGGTTGTAAATGG-3'.

\section{Statistical analysis}

The data were presented as the mean \pm SD. Student $t$ test and One-way ANOVA method followed by Bonferroni post hoc test were used to assess the difference between two groups and multiple groups, separately. A p $<0.05$ was considered statistically significant.

\section{Results \\ PRP reduced doxorubicin exposure-increased susceptibility to osteoarthritis}

To investigate whether PRP is able to exert a protective effect against doxorubicin-induced inflammation of chondrocytes, we treated mice with PRP and doxorubicin. As shown in Fig. 1A, B, doxorubicin treatment significantly degraded articular cartilage, and PRP injection protected mice against doxorubicin-induced osteoarthritis. In line with the pathological results, ELISA also revealed that doxorubicin exposure increased TNF- $\alpha$, IL-1 $\beta$, and IL6 protein levels, whereas PRP 

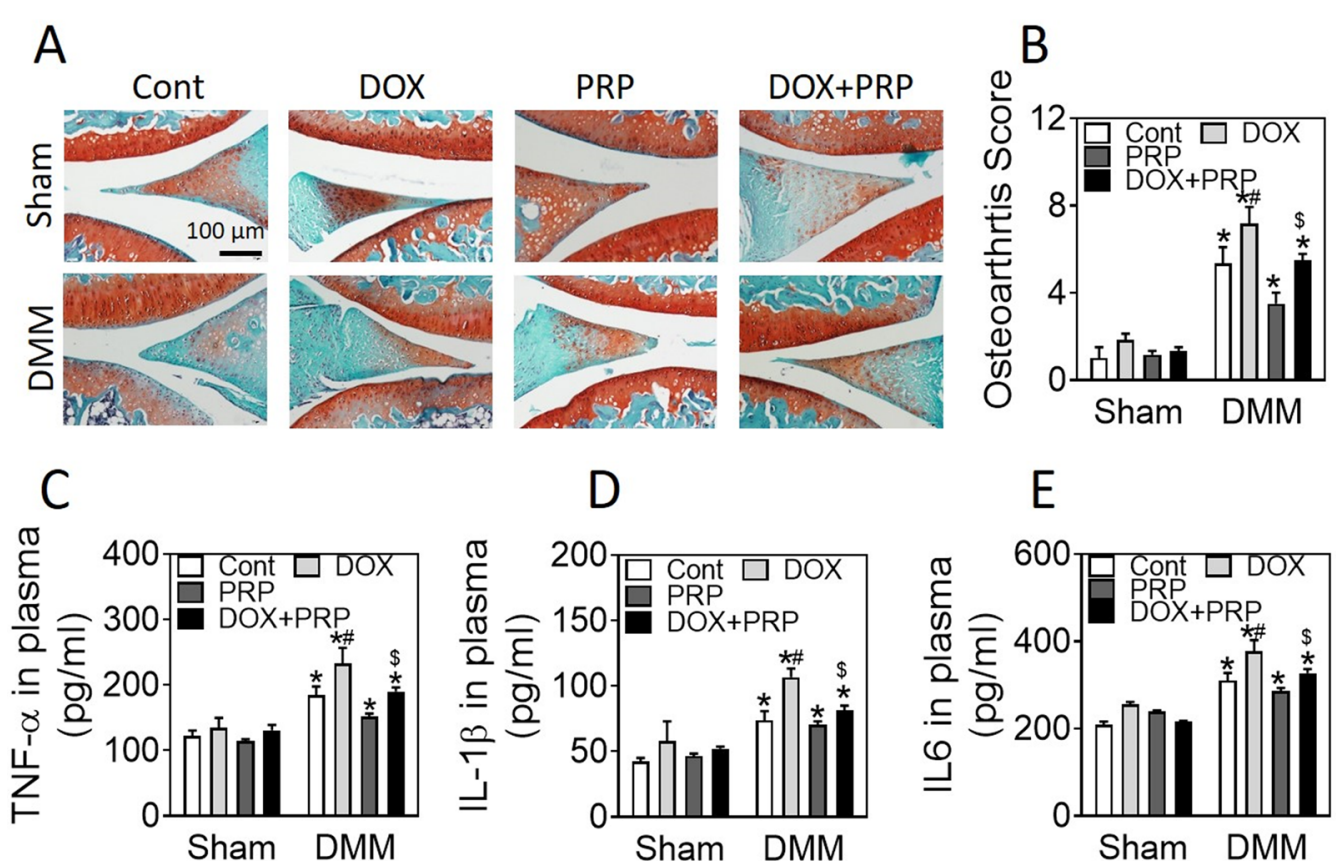

Fig. 1 PRP reduces doxorubicin exposure-increased susceptibility to osteoarthritis. The 12-week-old C57BL/6J mice were under DMM surgery for 1 week and received doxorubicin (10 mg/kg) injection and/or intra-articular injection of $15 \mu \mathrm{L}$ PRP every week. After 4 weeks, mouse knee joints were collected. A, B Representative images of Safranin O/fast green staining (A) and quantification (B). C-E Plasma levels of TNF- $a(\mathbf{C})$, IL-1 $\beta$ (D), and IL6 (E). $n=6 .{ }^{*} p<0.05$, vs sham of the same treatment; ${ }^{*} p<0.05$, vs control of the same DMM treatment; ${ }^{\$} p<0.05$, vs doxorubicin of the same DMM treatment

administration suppressed these increases (Fig. 1C-E). Together, these data suggested that PRP injection ameliorated doxorubicin exposure-induced osteoarthritis, probably through suppressing inflammation.

PRP inhibited doxorubicin-induced chondrocyte apoptosis As the extract from total blood, PRP is considered a cocktail of growth factors, among which TGF- $\beta 1$ is the most abundant, presentative ingredient. In this study, we determined PRP concentration by evaluating TGF- $\beta 1$ level and investigated the proliferation of ATDC5 cells and primary articular chondrocytes. The results showed that $2 \mathrm{ng} / \mathrm{mL}$ PRP treatment enhanced proliferation of ATDC5 cells (Fig. 2A) and primary articular chondrocytes (Fig. 2D) compared with the control and lower concentration PRP treatment. We next evaluated the potential of doxorubicin to affect cell viability and apoptotic cell death. As shown in Fig. 2B, E, treatment with lower than $40 \mu \mathrm{M}$ doxorubicin for $24 \mathrm{~h}$ had no significant effect on the viability of ATDC5 cells (Fig. 2B) and primary articular chondrocytes (Fig. 2E) compared with the control group. Hence, the maximum doxorubicin concentration used for subsequent experiments was $40 \mu \mathrm{M}$. We treated cells with doxorubicin and different concentrations of PRP for $24 \mathrm{~h}$, and found that doxorubicin treatment increased the apoptosis-related marker PARP and cleaved-PARP, and PRP treatment inhibited doxorubicin-induced PARP cleavage in ATDC5 (Fig. 2C) and primary articular chondrocytes (Fig. 2F). These results suggested that PRP inhibited doxorubicin-induced chondrocyte apoptosis.

\section{PRP suppressed doxorubicin-induced inflammation}

To investigate the role of PRP on inflammation, we examined cell matrix degradation and levels of several critical inflammatory factors. As shown in Fig. 3A, doxorubicin exposure for $24 \mathrm{~h}$ notably suppressed the levels of SOX9, Col2A1, Col10A1, and Aggrecan, whereas this suppression was attenuated by PRP at a dose-dependent manner (Fig. 3A, B, p < 0.05). As expected, PRP treatment reversed elevated levels of MMP-1, MMP-3, and MMP-13, the potent enzymes for cartilage matrix degradation compared with doxorubicin treatment alone (Fig. 3B, p<0.05). Furthermore, doxorubicin exposure increased the levels of inflammatory factors COX-2 and iNOS (Fig. $3 \mathrm{C}, \mathrm{p}<0.05$ ) and other inflammatory indicators, such as TNF- $\alpha$ and IL-6 (Fig. 3D, $\mathrm{p}<0.05$ ). Moreover, PRP inhibited the effects of doxorubicin on these inflammatory biomarkers (Fig. 3D, $\mathrm{p}<0.05$ ). These data indicate that PRP could improve chondrocyte damage via suppressing inflammatory biomarkers elevated by doxorubicin exposure. 


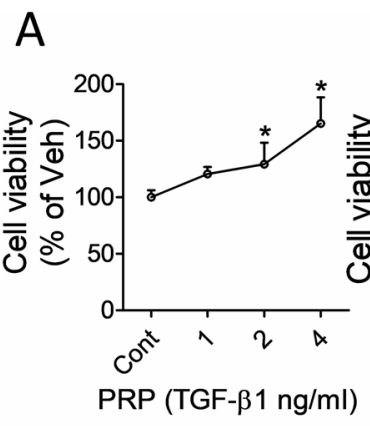

D

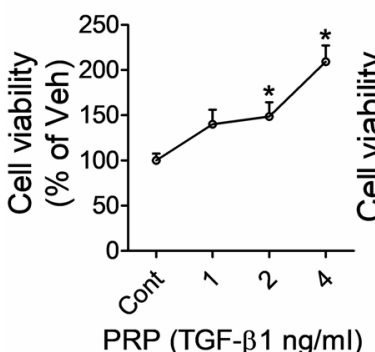

B

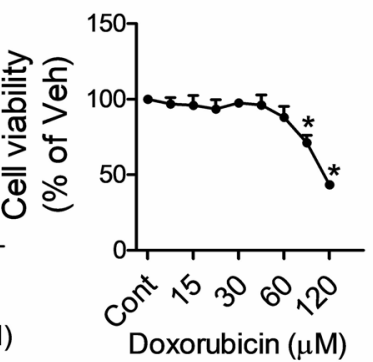

E

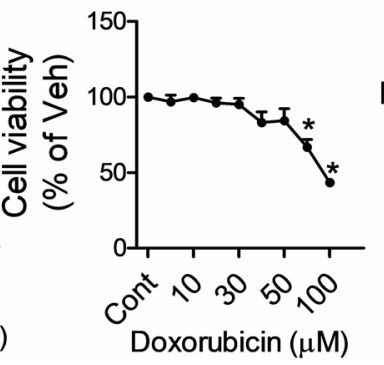

C

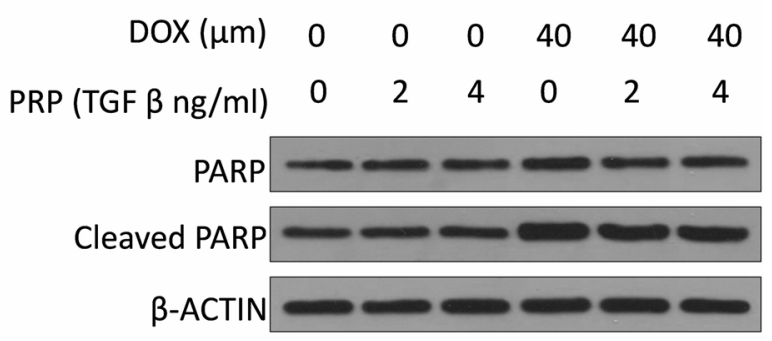

$\mathrm{F}$

$\operatorname{DOX}(\mu \mathrm{m}) \quad 0 \quad 0 \quad 0 \quad 0 \quad 40 \quad 40 \quad 40$

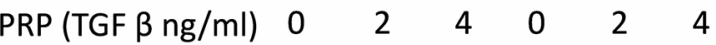

PARP $-\infty-\infty-\infty$

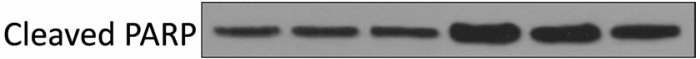

$\beta$-ACTIN

Fig. 2 PRP inhibits doxorubicin-induced chondrocyte apoptosis. ATDC5 cells and primary articular chondrocytes were treated with elevated concentrations of PRP and 40 doxorubicin for 24 h. A, B CCK-8 assay to determine ATDC5 viability after PRP (A) or doxorubicin (B) treatment. C Western blot assay to detect protein levels of PARP and cleaved PARP in ATDC5 cells. D, E CCK-8 assay to determine the viability of primary articular chondrocyte cells treated with PRP (D) or doxorubicin (E). F Western blot assay to detect protein levels of PARP and cleaved PARP in primary articular chondrocyte cells. At least three independent experiments were performed. ${ }^{*} p<0.05$ vs control

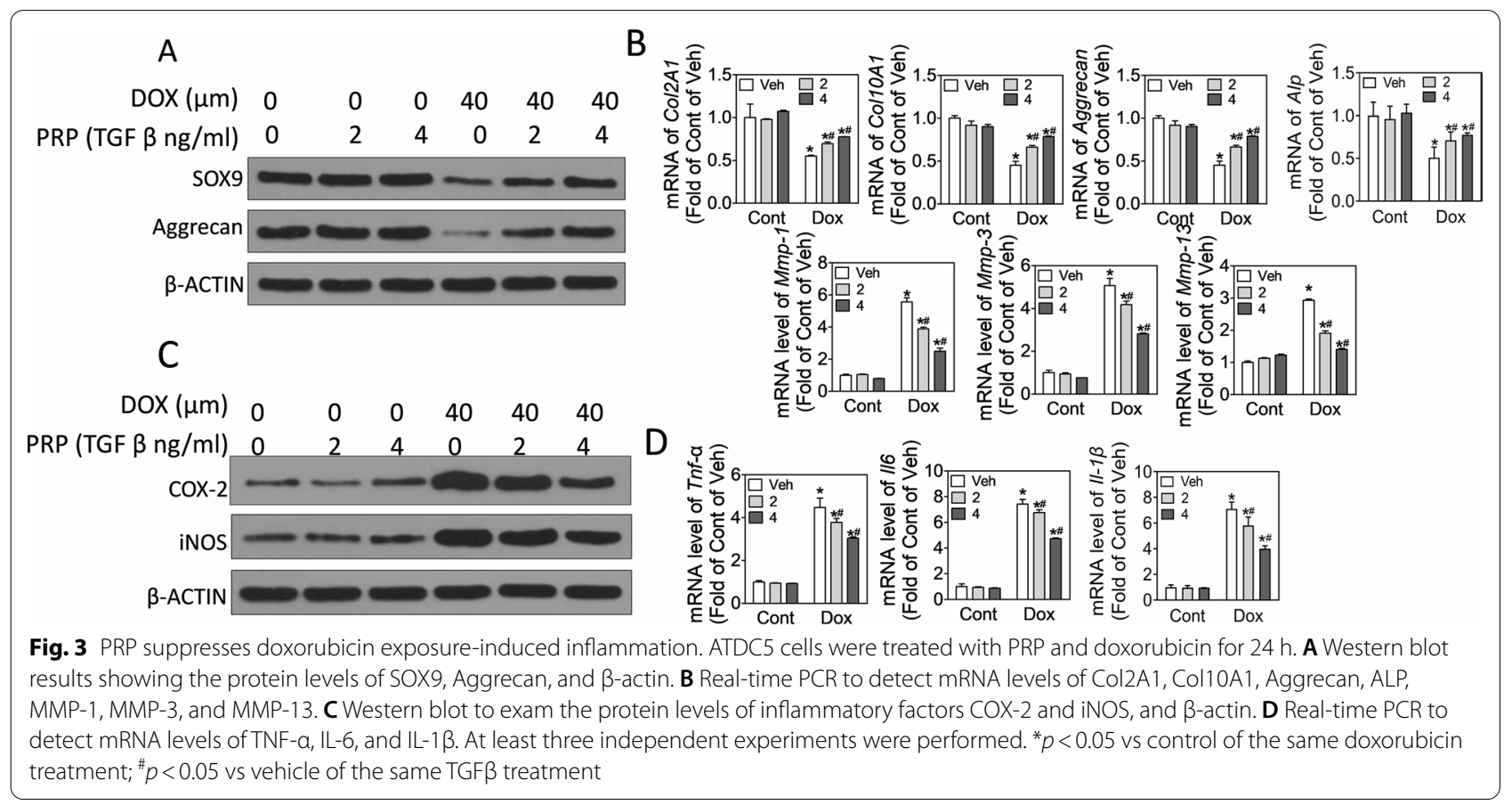




\section{PRP inhibited doxorubicin-induced inflammation via blocking NF-kB pathway}

To further elucidate mechanisms underlying PRP-suppressed inflammation under doxorubicin administration, we detected the NF-kB signaling pathway. The results showed that exposure to $40 \mu \mathrm{M}$ doxorubicin activated NF- $\kappa B$ pathway, with notably elevated I $\mathrm{B} \alpha$ phosphorylation and NF- $\mathrm{B}$ translocation to the nucleus, and these changes were suppressed by PRP in a dose-dependent manner (Fig. 4A-D, p < 0.05) in ATDC5. Moreover, PRP also reversed doxorubicin-induced NF- $\mathrm{KB}$ activation and elevated IL-6, IL-1 $\beta$ and TNF- $\alpha$ levels in BMMs (Fig. 4EG). Hence, we hypothesized PRP protected chondrocytes from doxorubicin-induced inflammation by inhibiting NF- $\mathrm{kB}$ signaling pathway. We further explored whether the NF-kB-dependent regulatory axis is also involved in the protective effects of PRP on primary articular chondrocyte cells. Consistent with the results from ATDC5, PRP remarkably reversed doxorubicin-elevation of inflammatory factors through suppressing NF- $\mathrm{kB}$ signaling activation (Fig. 5A, B, p <0.05). Besides, PRP treatment dose-dependently ameliorated (Fig. 5C, p<0.05) doxorubicin-induced cartilage matrix degradation, as manifested by reducing doxorubicin-induced elevation of MMP-1, MMP-3, MMP-13, and inflammatory factors TNF- $\alpha$ and IL- 6 in primary articular chondrocytes cells (Fig. 5C, p<0.05). In addition, because PRP contains many growth factors with the most abundant TGF$\beta 1$, we wondered whether TGF- $\beta 1$ alone is enough to explain the effects of PRP on chondrocytes. Therefore, we evaluated the effects of TGF- $\beta 1$ on the PARP cleavage and NF- $\mathrm{KB}$ activation. The results showed that TGF- $\beta 1$ blocked PARP cleavage and NF- $\mathrm{kB}$ activation in ATDC5, SW1353, and BMMs (Additional file 2: Fig. S2), indicating that PRP inhibits doxorubicin-induced inflammation via blocking NF-kB pathways.

\section{Discussion}

As a potent anti-cancer medicine, doxorubicin was widely used in clinical studies. However, its side effects severely restricted its application (Speth et al. 1988; Rivankar 2014). Numerous studies have indicated doxorubicin could cause normal cell death in various cell types, including chondrocytes (Kumagai et al. 2012). Our study also showed that doxorubicin treatment notably decreased the thickness of articular cartilage in the DMM model and elevated chondrocyte apoptosis and cleaved PARP protein level in vitro. These data confirmed

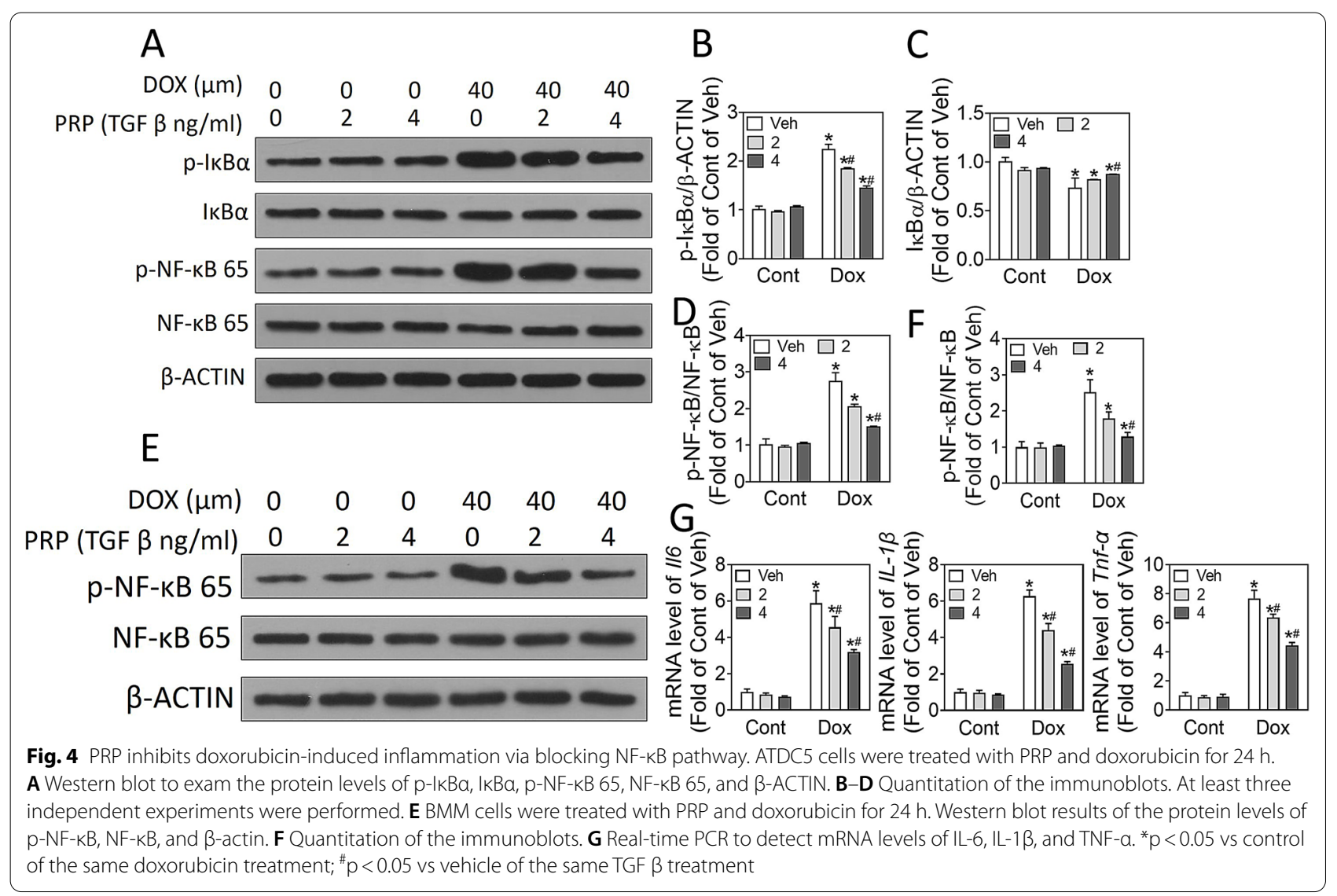




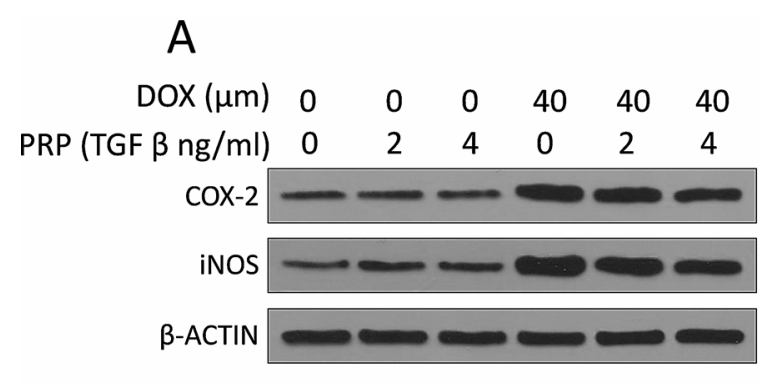

C

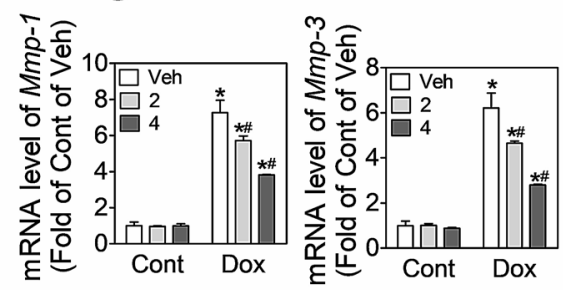

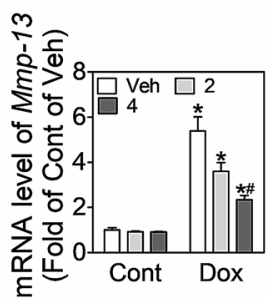

$\mathrm{B}$ DOX $(\mu \mathrm{m}) \quad 0 \quad 0 \quad 0040 \quad 40 \quad 40$

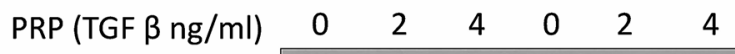

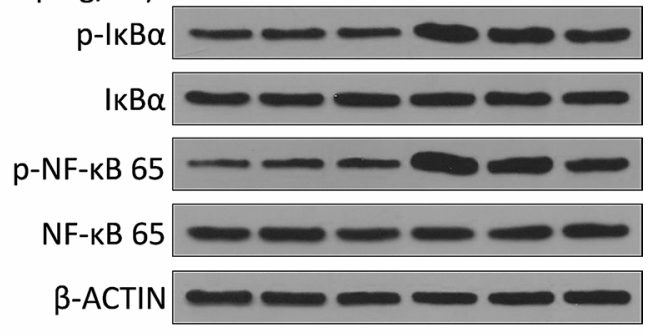

Fig. 5 PRP inhibits doxorubicin-induced inflammation in SW1353. SW1353 were treated with PRP and doxorubicin for $24 \mathrm{~h}$. A, B Western blot to detect protein levels of COX-2, iNOS, p-IKBa, IKBa, p-NF-kB 65, NF-kB 65, and $\beta$-actin. C mRNA levels of MMP-1, MMP-3, MMP-13, TNF-a, and IL-6. At least three independent experiments were performed. ${ }^{*} p<0.05 \mathrm{vs}$ control of the same doxorubicin treatment; $\# p<0.05$ vs vehicle of the same TGF $\beta$ treatment

the apoptosis-promoting role of doxorubicin during OA progression.

Early studies on OA stated that cartilage degradation could be independent of inflammatory response. However, numerous studies have proposed that low-grade and chronic inflammation at early stage remarkably promotes OA progression (Robinson et al. 2016; Chang et al. 2019; Shen et al. 2017). Hence, more therapeutic approaches are being developed to directly target the components of inflammatory signaling, among which the intra-articular PRP injection seems to be accompanied with much favorable outcomes (Fotouhi et al. 2018; Smith 2016; Xie et al. 2015).

The therapeutic effects of PRP on OA are considered mainly mediated by the anabolic growth factors and antiinflammatory mediators through activating collagen synthesis in chondrocytes and simultaneously preventing chondrocyte apoptosis (Kennedy et al. 2018). Our study suggested that PRP injection notably attenuated doxorubicin-caused cartilage degeneration in the DMM model and suppressed levels of inflammatory factors TNF$\alpha$, IL- $1 \beta$, and IL- 6 . We found that $40 \mu \mathrm{M}$ doxorubicin treatment did not affect cell viability. Thus, it was used in subsequent in vitro studies. In vitro experiments further revealed PRP administration suppressed Cox 2 and iNOS expression in doxorubicin-treated chondrocytes, consistent with our previous results that doxorubicininduced cell apoptosis is associated with reactive oxygen species (ROS) production, MMP gene expression, and superoxide generation. Administration of PARP inhibitors and oxidase inhibitors could attenuate doxorubicininduced cell death (Kalivendi et al. 2001; Mukhopadhyay et al. 2009; Mizutani et al. 2005). It has been reported that protein degradation induced by ubiquitin ligases is important in this system (Brigant et al. 2020). We, therefore, speculated that ubiquitin ligases might be involved in the underlying mechanism for apoptosis in chondrocytes induced by doxorubicin.

Sox9, Aggrecan, and type II collagen are produced by normal chondrocytes to facilitate chondrogenesis in joints and are regarded as particular chondrogenic

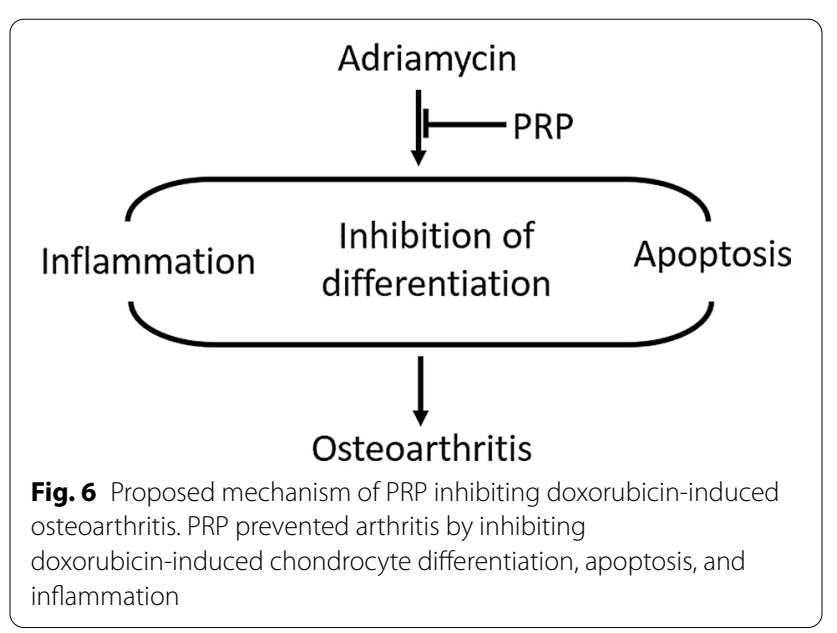


biomarkers (Carballo et al. 2017). SOX9 functions as a crucial transcriptional factor at the early stage of chondrogenesis and is capable of activating the expression of specific cellular matrix proteins, including type II collagen (Col2A) and Aggrecan (Sanchez et al. 2017; Henrotin et al. 2007; Rousseau and Garnero 2012). These proteins exert important functions to maintain the biological structure of articular cartilage (Gu et al. 2014). Here, we found decreased expression of SOX9 and Aggrecan and impaired transcription of Col2A1, Col10A1 and Aggrecan genes under doxorubicin treatment. However, PRP could notably ameliorate the damaged matrix proteins, which further supported the favorable function of PRP in OA therapy.

$\mathrm{NF}-\mathrm{kB}$ is a classic transcriptional factor during numerous inflammatory responses in multiple diseases, including OA (Saito and Tanaka 2017). Previous studies have revealed that NF- $k B$ signaling upregulates the expression of MMPs, the enzymes that directly degrade collagens, leading to chondrocyte hypertrophy and formation of inflammatory OA (Lepetsos et al. 2019). $N F-\kappa B$ signaling is also critical for the expression of various inflammatory factors, including iNOS, TNF- $\alpha$, and ILs (Liu et al. 2017). Our in vitro and in vivo studies showed that PRP administration notably reversed doxorubicin-induced elevation of inflammatory factors and collagen degradation during OA. We wonder if these anti-inflammatory functions of PRP are associated with $\mathrm{NF}-\mathrm{KB}$ signaling. As expected, in both primary chondrocytes and ATDC5 cells, PRP treatment significantly suppressed doxorubicin-activated ІкB phosphorylation and NF- $\mathrm{KB}$, which could inhibit NF- $\mathrm{KB}$ translocation from cytoplasm to nucleus and expression of various inflammatory factors.

\section{Conclusion}

In conclusion, our study showed that the platelet-rich plasma (PRP) collected from blood exhibited favorable effects against doxorubicin-caused damages to articular cartilage in vivo. PRP suppressed doxorubicin-induced chondrocyte apoptosis and matrix degradation by attenuating NF- $\mathrm{kB}$ signaling and expression of downstream inflammatory factors iNOS, TNF- $\alpha$, and ILs (Fig. 6). Our findings may provide more supportive evidence for the application of PRP to OA therapy.

\section{Supplementary Information}

The online version contains supplementary material available at https://doi. org/10.1186/s10020-021-00314-2.

Additional file 1: Figure S1. Toluidine blue staining and collagen type II immunohistochemical staining of the mouse chondrocytes.
Additional file 2: Figure S2. TGF- $\beta 1$ is the crucial factor in PRP responsible for its preventive effects on arthritis. Shown are the Western blot results of PARP, cleaved PARP, $\beta$-actin, p-NF-KB, and NF-KB in ATDC5 (A), primary articular chondrocytes cells (C), SW1353 (E) and BMMs (H) treated with PRP, doxorubicin, and TGF- $\beta 1$ for $24 \mathrm{~h}, \%$ of PRP action is the effect of TGF- $\beta 1$ and other in ATDC5 (B), primary articular chondrocytes cells (D), SW1353 (F) and BMMs (H).

\section{Acknowledgements}

Not applicable.

\section{Authors' contributions}

HZ: study concepts, literature research, clinical studies, data analysis, experimental studies, manuscript writing and review; WZ: study design, literature research, experimental studies and manuscript editing;WM: definition of intellectual content, clinical studies, data acquisition and statistical analysis; CS: data acquisition, manuscript preparation and data analysis. All authors read and approved the final manuscript.

\section{Funding}

Not applicable.

\section{Availability of supporting data}

The data that support the findings of this study are available on request from the corresponding author Haijun Zhao, Department of Joint Trauma Surgery, Qingdao Jiaozhou Central Hospital, No. 29 Xuzhou Road, Jiaozhou City, Shandong Province, 266300, P. R. China. Email: HaijunZhaoJiaozhou@163.com. The data are not publicly available due to their containing information that could compromise the privacy of research participants.

\section{Declarations}

Ethics approval and consent to participate

Informed consent was obtained from all individual participants included in the study. All producers were approved by the Ethics Committee of Qingdao Jiaozhou Central Hospital.

\section{Consent to publish}

Not applicable.

\section{Competing interests}

All authors have no conflicts of interest.We declare that we do not have any commercial or associative interest that represents a conflict of interest in connection with the work submitted.

Received: 24 October 2020 Accepted: 19 May 2021 Published online: 25 June 2021

\section{References}

Bannuru RR, Schmid CH, Kent DM, Vaysbrot EE, Wong JB, McAlindon TE. Comparative effectiveness of pharmacologic interventions for knee osteoarthritis: a systematic review and network meta-analysis. Ann Intern Med. 2015;162(1):46-54.

Bos-Mikich A, de Oliveira R, Frantz N. Platelet-rich plasma therapy and reproductive medicine. J Assist Reprod Genet. 2018;35(5):753-6.

Brigant B, Demont Y, Ouled-Haddou H, Metzinger-Le Meuth V, Testelin S, Garcon L, et al. TRIM37 is highly expressed during mitosis in CHON-002 chondrocytes cell line and is regulated by miR-223. Bone. 2020;137:115393.

Carballo CB, Nakagawa Y, Sekiya I, Rodeo SA. Basic science of articular cartilage. Clin Sports Med. 2017;36(3):413-25.

Chang SH, Mori D, Kobayashi H, Mori Y, Nakamoto H, Okada K, et al. Excessive mechanical loading promotes osteoarthritis through the gremlin-1-NFkappaB pathway. Nat Commun. 2019;10(1):1442.

Chen D, Shen J, Zhao W, Wang T, Han L, Hamilton JL, et al. Osteoarthritis: toward a comprehensive understanding of pathological mechanism. Bone Res. 2017;5:16044. 
Chouhan DK, Dhillon MS, Patel S, Bansal T, Bhatia A, Kanwat H. Multiple platelet-rich plasma injections versus single platelet-rich plasma injection in early osteoarthritis of the knee: an experimental study in a guinea pig model of early knee osteoarthritis. Am J Sports Med. 2019;47(10):2300-7.

Fotouhi A, Maleki A, Dolati S, Aghebati-Maleki A, Aghebati-Maleki L. Platelet rich plasma, stromal vascular fraction and autologous conditioned serum in treatment of knee osteoarthritis. Biomed Pharmacother. 2018;104:652-60.

Fukawa T, Yamaguchi S, Akatsu Y, Yamamoto Y, Akagi R, Sasho T. Safety and efficacy of intra-articular injection of platelet-rich plasma in patients with ankle osteoarthritis. Foot Ankle Int. 2017;38(6):596-604.

Gregori D, Giacovelli G, Minto C, Barbetta B, Gualtieri F, Azzolina D, et al. Association of pharmacological treatments with long-term pain control in patients with knee osteoarthritis: a systematic review and meta-analysis. JAMA. 2018;320(24):2564-79.

Gu J, Lu Y, Li F, Qiao L, Wang Q, Li N, et al. Identification and characterization of the novel Col10a1 regulatory mechanism during chondrocyte hypertrophic differentiation. Cell Death Dis. 2014;5:e1469.

Guilak F, Nims RJ, Dicks A, Wu CL, Meulenbelt I. Osteoarthritis as a disease of the cartilage pericellular matrix. Matrix Biol. 2018;71-72:40-50.

Henrotin Y, Addison S, Kraus V, Deberg M. Type II collagen markers in osteoarthritis: what do they indicate? Curr Opin Rheumatol. 2007;19(5):444-50.

$\mathrm{Hu}$ J, Zhou J, Wu J, Chen Q, Du W, Fu F, et al. Loganin ameliorates cartilage degeneration and osteoarthritis development in an osteoarthritis mouse model through inhibition of NF-kappaB activity and pyroptosis in chondrocytes. J Ethnopharmacol. 2020;247:112261.

Kalivendi SV, Kotamraju S, Zhao H, Joseph J, Kalyanaraman B. Doxorubicininduced apoptosis is associated with increased transcription of endothelial nitric-oxide synthase. Effect of antiapoptotic antioxidants and calcium. J Biol Chem. 2001;276(50):47266-76.

Kennedy MI, Whitney K, Evans T, LaPrade RF. Platelet-rich plasma and cartilage repair. Curr Rev Musculoskelet Med. 2018;11(4):573-82.

Kumagai K, Imai S, Toyoda F, Okumura N, Isoya E, Matsuura H, et al. 17betaOestradiol inhibits doxorubicin-induced apoptosis via block of the volume-sensitive $\mathrm{Cl}(-)$ current in rabbit articular chondrocytes. $\mathrm{Br}$ J Pharmacol. 2012;166(2):702-20.

Lepetsos P, Papavassiliou KA, Papavassiliou AG. Redox and NF-kappaB signaling in osteoarthritis. Free Radic Biol Med. 2019;132:90-100.

Liao L, Zhang S, Gu J, Takarada T, Yoneda Y, Huang J, et al. Deletion of Runx2 in articular chondrocytes decelerates the progression of DMM-induced osteoarthritis in adult mice. Sci Rep. 2017;7(1):2371.

Liu T, Zhang L, Joo D, Sun SC. NF-kappaB signaling in inflammation. Signal Transduct Target Ther. 2017;2:17023.
Mizutani H, Tada-Oikawa S, Hiraku Y, Kojima M, Kawanishi S. Mechanism of apoptosis induced by doxorubicin through the generation of hydrogen peroxide. Life Sci. 2005;76(13):1439-53.

Mukhopadhyay P, Rajesh M, Batkai S, Kashiwaya Y, Hasko G, Liaudet L, et al. Role of superoxide, nitric oxide, and peroxynitrite in doxorubicininduced cell death in vivo and in vitro. Am J Physiol Heart Circ Physiol. 2009;296(5):H1466-83.

Rivankar S. An overview of doxorubicin formulations in cancer therapy. J Cancer Res Ther. 2014;10(4):853-8.

Robinson WH, Lepus CM, Wang Q, Raghu H, Mao R, Lindstrom TM, et al. Lowgrade inflammation as a key mediator of the pathogenesis of osteoarthritis. Nat Rev Rheumatol. 2016;12(10):580-92.

Rousseau J, Garnero P. Biological markers in osteoarthritis. Bone. 2012;51(2):265-77.

Saito T, Tanaka S. Molecular mechanisms underlying osteoarthritis development: Notch and NF-kappaB. Arthritis Res Ther. 2017;19(1):94.

Sanchez C, Bay-Jensen AC, Pap T, Dvir-Ginzberg M, Quasnichka H, Barrett-Jolley $\mathrm{R}$, et al. Chondrocyte secretome: a source of novel insights and exploratory biomarkers of osteoarthritis. Osteoarthr Cartil. 2017;25(8):1199-209.

Shen J, Abu-Amer Y, O'Keefe RJ, McAlinden A. Inflammation and epigenetic regulation in osteoarthritis. Connect Tissue Res. 2017;58(1):49-63.

Sherwood J. Osteoarthritis year in review 2018: biology. Osteoarthr Cartil. 2019;27(3):365-70.

Smith PA. Intra-articular autologous conditioned plasma injections provide safe and efficacious treatment for knee osteoarthritis: an FDA-sanctioned, randomized, double-blind, placebo-controlled clinical trial. Am J Sports Med. 2016;44(4):884-91.

Speth PA, van Hoesel QG, Haanen C. Clinical pharmacokinetics of doxorubicin. Clin Pharmacokinet. 1988;15(1):15-31.

Xie X, Zhang C, Tuan RS. Biology of platelet-rich plasma and its clinical application in cartilage repair. Arthritis Res Ther. 2014;16(1):204.

Xie X, Ulici V, Alexander PG, Jiang Y, Zhang C, Tuan RS. Platelet-rich plasma inhibits mechanically induced injury in chondrocytes. Arthroscopy. 2015;31(6):1142-50.

Xu Z, Luo J, Huang X, Wang B, Zhang J, Zhou A. Efficacy of platelet-rich plasma in pain and self-report function in knee osteoarthritis: a best-evidence synthesis. Am J Phys Med Rehabil. 2017;96(11):793-800.

\section{Publisher's Note}

Springer Nature remains neutral with regard to jurisdictional claims in published maps and institutional affiliations.
Ready to submit your research? Choose BMC and benefit from:

- fast, convenient online submission

- thorough peer review by experienced researchers in your field

- rapid publication on acceptance

- support for research data, including large and complex data types

- gold Open Access which fosters wider collaboration and increased citations

- maximum visibility for your research: over $100 \mathrm{M}$ website views per year

At BMC, research is always in progress.

Learn more biomedcentral.com/submissions 